\title{
Erratum to: Curative Approach for Stage IV Colorectal Cancer with Multiorgan Involvement: What Makes Sense and What Doesn't?
}

Polina Khrizman • Mary F. Mulcahy

Published online: 1 May 2010

(C) Springer Science+Business Media, LLC 2010

Erratum to: Curr Colorectal Cancer Rep

DOI 10.1007/s11888-010-0050-5

The original version of this article unfortunately contained incorrect data on Table 1. The correct Table 1 is shown here.

The online version of the original article can be found at http://dx.doi. org/10.1007/s11888-010-0050-5.

P. Khrizman $\cdot$ M. F. Mulcahy $(\triangle)$

Northwestern University Feinberg School of Medicine,

676 North St. Clair, Suite 850,

Chicago, IL 60611, USA

e-mail: m-mulcahy@northwestern.edu

P. Khrizman

e-mail: polina.khrizman@gmail.com 
Table 1 Studies with synchronous or metachronous metastatic colorectal cancer resection to the liver and lung

\begin{tabular}{|c|c|c|c|c|c|c|c|}
\hline \multirow[t]{2}{*}{ Author } & \multirow[t]{2}{*}{ Year } & \multirow[t]{2}{*}{ Patient, $\mathrm{n}$} & \multicolumn{3}{|l|}{ 5-year OS } & \multirow{2}{*}{$\begin{array}{l}10 \text {-year } \\
\text { OS, } \%\end{array}$} & \multirow{2}{*}{$\begin{array}{l}\text { Significant prognostic factors } \\
\text { (decreased/increased survival) }\end{array}$} \\
\hline & & & $\begin{array}{l}\text { Initial } \\
\text { surgery } \\
(\%)\end{array}$ & $\begin{array}{l}\text { First } \\
\text { metastasectomy } \\
(\%)\end{array}$ & $\begin{array}{l}\text { Last } \\
\text { mestastasectomy } \\
(\%)\end{array}$ & & \\
\hline $\begin{array}{l}\text { Regnard et al. } \\
\text { [20] }\end{array}$ & 1998 & 43 & 11 & NL & NL & NL & $\begin{array}{l}\text { (DS) CEA level }>5 \mathrm{ng} / \mathrm{mL} \\
\text { (DS) No. of pulmonary resections }=1 \\
\text { (DS) Interval between hepatic and } \\
\text { pulmonary resections }<36 \text { mo }\end{array}$ \\
\hline $\begin{array}{l}\text { Murata et al. } \\
\text { [21] }\end{array}$ & 1998 & 30 & 43.8 & NL & NL & NL & $\begin{array}{l}\text { (DS) Synchronous metastases } \\
\text { (DS) Bilateral pulmonary metastsases }\end{array}$ \\
\hline $\begin{array}{l}\text { Lehnert et al. } \\
\text { [24] }\end{array}$ & 1999 & 17 & 60 & 39 & NL & 43 and $20^{\mathrm{a}}$ & NL \\
\hline $\begin{array}{l}\text { Robinson et al. } \\
{[30]}\end{array}$ & 1999 & $\begin{array}{l}48 \\
\text { R: } 25 \\
\text { NR: } 23\end{array}$ & $\begin{array}{l}43 \\
0\end{array}$ & NL & $\begin{array}{l}9 \\
0\end{array}$ & NL & $\begin{array}{l}\text { (IS) Young patients at diagnosis } \\
\text { (IS) Solitary liver metastasis } \\
\text { (IS) Metachronous disease with long DFI } \\
\text { (IS) Resection of liver and lung } \\
\text { metastasis }\end{array}$ \\
\hline $\begin{array}{l}\text { Kobayashi et al. } \\
\text { [23] }\end{array}$ & 1999 & 47 & 31 & 22 and $50^{\mathrm{b}}$ & 40 and $22^{c}$ & NL & $\begin{array}{l}\text { (DS) Multiple pulmonary metastases-FM } \\
\text { and LM } \\
\text { (DS) Synchronous hepatic and pulmonary } \\
\text { metastases-FM }\end{array}$ \\
\hline $\begin{array}{l}\text { Headrick et al. } \\
\text { [28] }\end{array}$ & 2001 & 58 & NL & 30 & 43 & 16 & $\begin{array}{l}\text { (DS) CEA level }>5 \mathrm{ng} / \mathrm{mL} \\
\text { (DS) Thoracic } \mathrm{LN} \text { metastases }\end{array}$ \\
\hline $\begin{array}{l}\text { Nagakura et al. } \\
\text { [22] }\end{array}$ & 2001 & 27 & 27 & 44 and $0^{d}$ & NL & NL & $\begin{array}{l}\text { (DS) Age at initial metastasectomy } \geq 60 \\
\text { (DS) Simultaneous detection of hepatic } \\
\text { and pulmonary metastases }\end{array}$ \\
\hline Mineo et al. [27] & 2003 & 29 & NL & 51.3 & NL & NL & (DS) Elevated CEA and CA 19-9 levels \\
\hline Shah et al. [29] & 2006 & 39 & 117 months $^{\mathrm{e}}$ & 74 & NL & NL & $\begin{array}{l}\text { No prognostic factors were identified } \\
\text { Timing of presentation of metastatic } \\
\text { disease was not a significant } \\
\text { predictor of OS }\end{array}$ \\
\hline Miller et al. [26] & 2007 & 131 & 65 & 49 & 31 & $\begin{array}{l}32,20 \text {, and } \\
19^{f}\end{array}$ & $\begin{array}{l}\text { (DS) Age }>55 \\
\text { (DS) }>1 \text { liver metastasis } \\
\text { (DS) DFI } 1 \text { st to } 2 \text { nd metastasis } \\
\text { appearance }<1 \text { year }\end{array}$ \\
\hline Neef et al. [25] & 2009 & 44 & 64 & 42 & 27 & NL & (DS) Lung as 1st metastatic site \\
\hline
\end{tabular}

$C E A$ carcinoembryonic antigen, $D F I$ disease-free interval, $D S$ or $I S$ Decreased/Increased Survival, FM first metastasectomy, $L M$ last metastasectomy, $L N$ lymph node, $M O$ months, $N L$ not listed, $N R$ only primary resected, pulmonary and hepatic metastases not resected, $O S$ overall survival, $R$ resected pulmonary and hepatic metastases

${ }^{\text {a }} 43 \%$ is the 10 -year survival after primary tumor resection, $20 \%$ is the 10 -year survival after first metastasectomy

${ }^{\mathrm{b}} 22 \%$ in patients with synchronous metastases, and $50 \%$ in patients with hepatic metastases detected before pulmonary metastases

${ }^{\mathrm{c}} 40 \%$ in patients with a solitary pulmonary metastasis and $22 \%$-in patients with multiple pulmonary metastases

d $44 \%$ in 17 patients with sequentially detected hepatic and pulmonary metastases; $0 \%$ in patients with simultaneously detected metastases

${ }^{\mathrm{e}}$ Median overall survival is provided, no 5-year OS, 5-year OS after last metastasectomy, or a 10-year survival is documented

${ }^{\mathrm{f}} 10$-year survival is given from the primary colorectal cancer resection, from the first metastastasectomy, from the last metastasectomy, respectively 